$\Rightarrow$ CELL DEATH

\title{
Telomere crisis activates autophagic death
}

\section{$c$}

autophagy-

deficient cells

... continued

to proliferate and bypassed

crisis

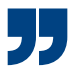

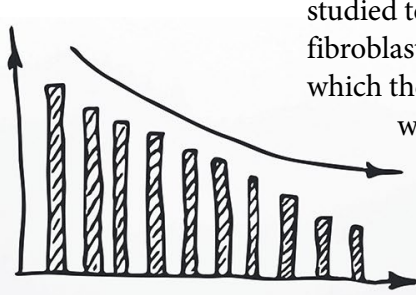

fibroblasts and epithelial cells, in which the RB and/or p53 pathways were suppressed; these cells bypassed senescence and entered replicative stress, exhibiting telomere attrition, chromosome fusions and cell death.

Cells in crisis had increased levels of autophagy proteins, including the autophagy protein 5 (ATG5)-

ATG12 conjugate and LC3-II (the lipidated form of light

chain 3 , which is the key

regulator of autophagosome biogenesis) and decreased levels of the cargo receptor p62 (also known as sequestosome 1), all indicative of enhanced autophagy.

Autophagy was directly monitored using a tandem fluorescent LC3 reporter. Similarly to cells in starvation, which are known to activate autophagy, cells in crisis accumulated multiple LC3-containing autophagosomes and autolysosomes. Inhibition of autophagic flux (progression) led to even greater accumulation of autophagosomes, but abolished autolysosomes. Thus, cells in replicative crisis activate autophagy.

Next, the authors tested whether suppression of autophagy enables cells to bypass crisis and evade death. Whereas cell death was frequent in control cells undergoing replicative crisis, autophagy-deficient cells (depleted of ATG3, ATG5 or ATG7) continued to proliferate and bypassed crisis. Cells that bypassed crisis accumulated nontelomeric double-strand breaks and gross chromosomal aberrations, demonstrating that the elimination of cells in crisis involves autophagy and is essential for the maintenance of genome integrity.

To compare the cellular response to telomeric breaks with that to intrachromosomal breaks, telomeric breaks were specifically induced by a fusion protein consisting of telomeric repeat-binding factor 1 (TRF1) and the restriction endonuclease FokI (TRF1-FokI). Whereas intrachromosomal breaks activated apoptosis, telomere damage led only to activation of autophagy.

Dicentric chromosomes formed by telomere fusion can fragment and release DNA into the cytosol. Indeed, cells in crisis, or growing cells depleted of the telomere protection protein TRF 2 or treated with TRF1-FokI exhibited a gradual increase in the frequency of nucleoplasmic chromatin bridges, micronuclei and cytoplasmic chromatin fragments; these were much less common in cells suffering from radiation-induced intrachromosomal breaks.

Telomere deprotection through TRF2 depletion was sufficient to activate autophagy independently of replicative crisis, and genetic suppression of telomere fusions in TRF2-depleted cells reduced the accumulation of cytosolic DNA and attenuated autophagy, suggesting that fusion-dependent cytosolic DNA is required for the telomeric autophagy response.

Cytoplasmic DNA triggers the cyclic GMP-AMP synthase (cGAS)-stimulator of interferon genes (STING) pathway, which can promote autophagy. In cells undergoing crisis, cytosolic DNA colocalized with cGAS, LC3 and p62. Moreover, cells lacking either cGAS or STING proliferated beyond crisis. Bypass of crisis involved attenuation of autophagy, as indicated by a decrease in LC3-II levels and an increase in p62 levels.

In summary, cells in telomere crisis undergo cell death through autophagy, which is triggered by chromosome breakage and transduced by the cGAS-STING pathway. As cell death in crisis represents the final barrier against neoplastic transformation, a cancer therapy that involves inhibition of autophagy could be counterproductive.

Eytan Zlotorynski

ORIGINAL ARTICLE Nassour, J. et al. Autophagic cell death restricts chromosomal instability during replicative crisis. Nature https://doi.org/10.1038/ 541586-019-0885-0 (2019) FURTHER READING Maciejowski, J. \& de Lange, T. Telomeres in cancer: tumour suppression and genome instability. Nat. Rev. Mol. Cell Biol. 18. 175-186 (2017)| Dikic, I. \& Elazar, Z. Mechanism and medical implications of mammalian autophagy. Nat. Rev. Mol. Cell Biol. 19, 349-364 (2018) 